\title{
TEMPORAL 3D SHAPE MATCHING
}

\author{
Peng Huang, Jonathan Starck and Adrian Hilton \\ Centre for Vision, Speech and Signal Processing \\ University of Surrey, Guildford, GU2 7XH, UK \\ \{P.Huang, J.Starck, A.Hilton\}@ surrey.ac.uk
}

Keywords: 3D Shape Similarity, Temporal 3D Shape Matching, Video-based Animation, 3D Video Texture

\begin{abstract}
This paper introduces a novel 4D shape descriptor to match temporal surface sequences. A quantitative evaluation based on the Receiver-Operator Characteristic (ROC) curve is presented to compare the performance of conventional 3D shape descriptors with and without using a time filter. Featurebased 3D Shape Descriptors including Shape Distribution [24], Spin Image [14], Shape Histogram [1] and Spherical Harmonics [16] are considered. Evaluation shows that filtered descriptors outperform unfiltered descriptors and the best performing volume-sampling shape-histogram descriptor is extended to define a new 4D "shape-flow" descriptor. Shape-flow matching demonstrates improved performance in the context of matching time-varying sequences which is motivated by the requirement to connect similar sequences for animation production. Both simulated and real 3D human surface motion sequences are used for evaluation.
\end{abstract}

\section{Introduction}

In animation synthesis, highly realistic content can be generated simply be re-organising recorded motion clips [27]. In this paper we concentrate on human surface motion [30, 34] in which a database of 3D time-varying geometry for different actions must be compared and concatenated at points where the similarity in 3D shape is maximised. Synthesis of new content requires a temporal descriptor to compare time-varying motion across different clips.

Video-based animation was first introduced by Schodl et al. [27] where video textures of arbitrary length are generated from captured video sequences. Similar frames must be found in a sequence such that the video-texture can loop seamlessly. Neumann et al. [21] note that actions are best defined as 4D patterns in space and time, and similarity should ideally be compared in spatio-temporal space. However, current matching techniques from the shape retrieval literature consider static shapes only $[31,6,12,13]$. Shapes at an instant in time can appear similar, but over time can belong to very different actions. For example, if a pendulum swinging from left to right is split into several frames, each one may be easily confused with a right to left swing. In such a case, temporal information must be added to resolve the ambiguity.
Spatio-temporal shape matching is a natural extension of current shape matching work. In [18] we reviewed and compared 3D shape descriptors from the shape retrieval literature for the problem of human surface shape similarity. The best performance was demonstrated by a shape histogram volume-based descriptor. In this paper, we extend previous shape descriptors by using a time filter to incorporate temporal information. Three contributions are made.

- A quantitative evaluation is presented against groundtruth data to compare the performance of conventional shape descriptors using a simple temporal filter.

- A new 4D histogram volume-based descriptor is introduced to define time-varying shape flow for a surface giving an improved temporal shape matching.

- Finally, the new shape-flow descriptor is demonstrated on real time-varying sequences of human motion.

In Section 2, shape descriptors for 3D shape retrieval are reviewed. Section 3 describes the shape descriptors evaluated and the construction of temporal descriptors using a time-filter and 4D shape flow. A quantitative evaluation is presented in section 4 using a synthetic ground-truth data-set. Performance is compared using the receiver-operator characteristic for the descriptors, the trade-off between correctly and incorrectly defined similarity. The performance is then demonstrated on real data and conclusions are finally drawn in Section 5.

\section{Background}

The problem of shape similarity has been widely studied in the 3D shape retrieval literature. There are several techniques: feature-based, graph-based, view-based and bending-invariant methods. Graph methods are based on matching surface topology and so do not necessarily handle changes in topology that can occur in captured sequences of articulated motion. View-based methods suffer from large changes in the projected shape of an object with only small changes in articulated pose. Bending-invariant methods explicitly match similarity independent of object deformations and so cannot differentiate time-varying sequences. Therefore, we only focus on featurebased methods here, and interested readers are referred to $[19,5]$ for a comprehensive review. Temporal methods from motion recognition and video texture are also reviewed for the problem of matching time-varying data. 


\subsection{Feature-based methods}

Feature-based methods are general and can be applied to any multimedia database. A feature describes a particular characteristic or set of characteristics for an object. Different algorithms capture different types of features, either global features or local features. The similarity between two objects is then defined by a distance measure between their features.

Global features characterise the overall shape of 3D objects, including volume, area, moments, Fourier and Wavelet coefficients. Zhang and Chen[36] efficiently calculate these features directly from a surface mesh representation. Paquet et al.[25] describe a representation for the coarse shape, scale and composition properties of an object, which is invariant to resolution, translation and rotation. Corney et al. [8] coarsely filter candidates in 3D model retrieval prior to a more detailed analysis and use convex-hull based indices. Kazhadan et al.[15] extract the global symmetry information to construct a reflective symmetry descriptor. These global features are relatively simple to compute but are relatively coarse.

Local features can give a more distinctive measure. Shum et al.[28] extract a local curvature distribution from a mesh representation and define a $L_{2}$ distance to measure similarity between two objects. Zaharia and Preteux [35] define the distribution of a shape index over the entire mesh as a 3D Shape Spectrum Descriptor (3D SSD), to provide an intrinsic 3D shape description. Chua and Jarvis [7] introduce a point signature, which is invariant to rotation and translation, to describe 3D free-form surfaces. Johnson and Hebert[14] use Spin Images in a 3D shape-based object recognition system. These features provide local shape information to improve discrimination between similar shapes.

Features can also be compared using their distribution. Osada et al.[24] presented a Shape Distribution descriptor, which measures global geometric properties of an object, to discriminate similar from dissimilar. The similarity measure is invariant to translation, rotation and tessellation. Ankerst et al.[1] use a 3D Shape Histogram descriptor, which is based on a partitioning of the space where an object resides, to classify a molecular database. Similarity is computed as the difference between histograms. Kortgen et al.[17] provide a 3D Shape Context descriptor to each surface sample point, which is the extension of a 2D Shape Context descriptor introduced by Belongie et al.[2] for 2D shape matching. Ohbuchi et al. [23] consider both the distance between point pairs and the angle formed by the surface normals to construct Angle Distance (AD) and Absolute Angle Distance (AAD) histograms for 3D shape matching.

Transform-based representations describe shapes in a transformation invariant manner. Ricard et al.[26] apply 3D Angular Radial Transform (3D-ART) on voxelized objects and use magnitudes of ART coefficients as descriptors. Vranic and Saupe [32] take the 3D Discrete Fourier Transform (3D-DFT) of the binary voxel representations after alignment to principal axes. Dutagaci et al. derive and compare two transform-based rotation invariant descriptors, namely Discrete Fourier Transform (DFT-based) and Radial Cosine Transform (RCT-based) descriptors in [10]. Kazhdan et al.[16] introduce Spherical Harmonic Representation as a 3D Shape Descriptor for 3D shape retrieval. Novotni and Klein [22] extended it to a 3D Zernike Descriptor. However, Spherical Harmonics and 3D Zernike suffer the same ambiguity problem: the descriptor is unchanged for objects whose frequency components are only different in rotation.

\subsection{Temporal methods}

There has been little previous work on temporal shape matching. But some similar ideas can be found in the Computer Graphics and Motion Recognition literature.

In Computer Graphics, Schodl et al.[27] introduced video textures which provide a continuous stream of images. They reuse the input video frames to synthesize a similar video of arbitrary length. Transition points are found by matching subsequences instead of individual frames in order to preserve the dynamics of motion. In practice, such a subsequence match is achieved by simply time filtering the frame-by-frame similarity matrix with a diagonal kernel. The similarity metrics are based on 2D image differences which cannot be directly extend to 3D time-varying surfaces.

In Motion Recognition, volumetric analysis of video, where a sequence of images is treated as a 3D space-time volume, is widely used. Video features are then extracted: Bobick et al.[4] combine Motion-Energy Images (MEI) and MotionHistory Images (MHI) as temporal motion templates for human movement recognition. Efros et al.[11] propose a pixel-wise optical-flow motion descriptor which is measured in a figure-centric spatio-temporal volume for each person, to obtain a motion-to-motion similarity matrix and time-filter the frame-to-frame similarities. Blank et al.[3] regard human actions as 3D shapes induced by the silhouettes in a spacetime volume, extracting features such as local space-time salience, action dynamics, shape structure and orientation. Weinland et al.[33] propose a free-viewpoint representation for human action based on a multi-camera system using Motion History Volumes (MHV) where alignment and comparison are performed under a Fourier transform in cylindrical coordinates around the vertical axis. These methods incorporate temporal information to achieve better recognition, which motivates us to extend 3D shape descriptors to 4D descriptors.

\section{Temporal 3D Shape Matching}

In this section, we first briefly describe Shape Descriptors used for $3 \mathrm{D}$ shape retrieval and their simple extension to temporal matching, using a time filter to incorporate temporal information. Then we introduce a temporal shape descriptor - the volume-based shape-flow histogram descriptor, for temporal 3D shape matching. 


\subsection{D Shape Descriptors}

Global features can only provide a coarse shape description that is insufficient to distinguish dissimilarity in a time varying sequence where an object can have very similar shape over a relatively long time period. Local feature based methods are therefore compared from the literature. Shape Distribution [24], Spin Image [14], Shape Histogram [1] and Spherical Harmonic [16] descriptors are implemented.

\subsubsection{Shape Distribution [24]}

A Shape Distribution computes the probability distribution of geometric properties of an object. Typical geometric properties include the angle, distance and area for random points on the surface. Here, we adopt the $D 2$ measure proposed by Osada et al. [24], which calculates the distribution of the distance between two random points on the surface. Similarity is then defined as the $L_{2}$ distance between the distributions. Given a $3 \mathrm{D}$ mesh representation the descriptor is constructed as follows:

- Distance is iteratively measured between two random points on the surface.

- A 1D histogram is created to count the number of pointpairs at different distances.

- The final histogram is normalised.

\subsubsection{Spin Image [14]}

A Spin Image is a 2D histogram which encodes the density of mesh vertices projected onto an object-centred space. Given a 3D surface mesh consisting of a set of oriented points corresponding to the mesh vertices, the histogram is constructed as follows:

- An object-centred coordinate $(\alpha, \beta)$ is computed for each vertex according to the distance $\alpha$ along and the distance $\beta$ from the principal axis of the object.

- A 2D accumulator indexed by $(\alpha, \beta)$ is created and the accumulator is incremented for each vertex within the support of the spin image.

- The final histogram is normalised.

The centre of mass and the first axis of the Principal Component Analysis (PCA) of the distribution of mesh vertices is used to define the object-centred coordinate system.

\subsubsection{Shape Histogram [1]}

A Shape Histogram partitions the space containing an object into disjoint cells corresponding to the bins of a histogram. Given a 3D surface mesh, a volume sampling spherical histogram is constructed as follows.

- A volumetric representation is constructed by rasterising the surface into a set of voxels that lie inside the model.

- Space is transformed to a spherical coordinate system $(r, \phi, \theta)$ around the centre of mass for the model.
- A 3D spherical histogram is constructed, accumulating the voxels in the volume representation.

- The final histogram is normalised.

The spherical coordinate histogram is compared invariant of rotation by testing similarity for all feasible rotations in $\phi$ and $\theta$. Instead of rotating a 3D mesh, we generate a fine histogram first, shift it with $1^{\circ}$ resolution, and re-bin to a coarse histogram. A similarity measure is computed as $L_{2}$ distance between the coarse histograms.

\subsubsection{Spherical Harmonics [16]}

A Spherical Harmonics Represention describes an object by a set of spherical basis functions. A descriptor is constructed by measuring the energy contained in different frequency bands, where the frequency components are rotation invariant. The descriptor is constructed as follows:

- The volume of an object is divided into a set of concentric shells.

- The frequency decomposition in each shell is computed directly from the mesh surface [20].

- The norm for each frequency component at each radius is concatenated into a 2D histogram indexed by radius and frequency.

The resolution of the shape descriptor is defined by the number of shells defining the radii $(r)$ and the preserved bandwidth (bw) in the spherical harmonics. A similarity measure is computed as $L_{2}$ distance between the histograms.

\subsection{Time-filtered Descriptors}

Time information can be incorporated in a static 3D shape descriptor using a temporal filter. Schodl et al. [27] and Efros et al. [11] use a similar strategy to achieve motion-to-motion matching. In practice, the time filter is applied to the frameto-frame similarity matrix obtained by 3D Shape Descriptors. Let $S_{f}(i, j)$ be the frame-to-frame similarity matrix, where $i$ and $j$ index the two sequences respectively in time order. A time filter $T\left(N_{t}\right)$ with window size $2 N_{t}+1$ is defind by $T\left(N_{t}\right)=1 /\left(2 N_{t}+1\right) * I$ where $I$ is an identity matrix. Temporal similarity matrix $S_{m}(i, j)$ is obtained by convoluting with $T\left(N_{t}\right), S_{m}=S_{f} \otimes T\left(N_{t}\right)$. We calculate the temporal similarity matrix as follows:

$$
S_{m}(i, j)=\frac{1}{2 N_{t}+1} \sum_{k=-N_{t}}^{N_{t}} S_{f}(i+k, j+k)
$$

The effect of time-filtering is illustrated in Figure 1. The figure shows self-similarity for a periodic motion as illustrated in earlier papers [9]. The ground-truth similarity matrix shows that the motion has a periodic similarity along the diagonal where time increases for the motions. The unfiltered results show an ambiguity with similar scores along the opposite diagonal, where time increases on one axis and decreases on the other. Forward and reverse motions in the sequence can be matched when considering only a single time instance. 


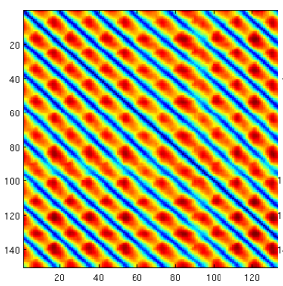

(a) Ground-Truth

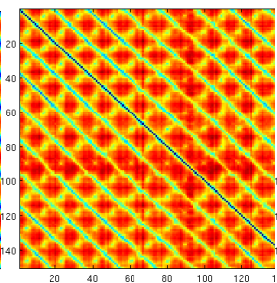

(b) Unfiltered

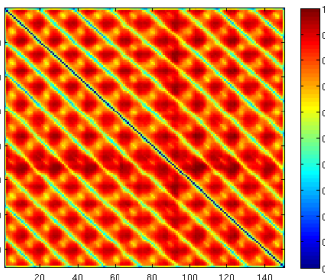

(c) Filtered

Figure 1: Similarity Matrix for the motion sprint. (a) Ground-truth similarity matrix; (b) Unfiltered Similarity Matrix obtained by the rotated volume-spherical Shape Histogram (SHvr) descriptor; (c) Filtered Similarity Matrix using a time window with size $3, N_{t}=1$.

After filtering, motions in the same direction are more similar. Note that some ambiguity can remain after time-filtering where the individual frames used in the filter are similar and the temporal information is limited. Although simply increasing time window size may help, a more distinctive temporal descriptor is now presented.

\subsection{Shape-Flow Descriptors}

A new 4D shape descriptor is introduced to define the change in shape for a surface in a subsequence corresponding to a given time window. Simply applying a time-filter to a static shape comparison breaks the temporal consistency in a motion as each static comparison is aligned independently. The new descriptor considers not only the similarity between individual frames in a subsequence but also preserves the temporal changes using a single subsequence alignment.

Previous work [18] has demonstrated that the volume sampling 3D spherical histogram gives the best performance in classifying shape similarity. The 3D histogram is extended here to incorporate changes in shape using a $4 \mathrm{D}$ histogram in which each 3D spherical bin records the shape over a 1D time window. Similarity is again defined using the $L_{2}$ distance between coarse histograms after alignment.

Histogram alignment is considered using two methods, either by finding the optimal alignment of the $3 \mathrm{D}$ descriptor for the centre frame of the temporal window, or by finding the optimal alignment of the entire sequence in the 4D descriptor. We call the first method single-frame shape-flow matching and the second group-frame shape-flow matching. A single-frame shape-flow matching has the same computational complexity with original shape matching but may not find the optimal alignment for the whole subsequence. A group-frame shapeflow matching is more robust but the computational cost is proportional to the time window size. The choice is a trade off between computational complexity and comparison accuracy. Both techniques are implemented for comparison.

Optimal alignment is derived first by finding the translation that matches the centre of mass and then by a direct search for the rotation that gives the greatest similarity between

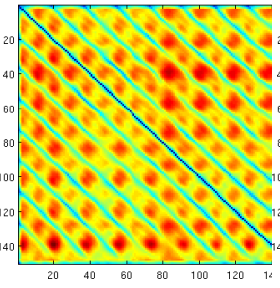

(a) Filtered

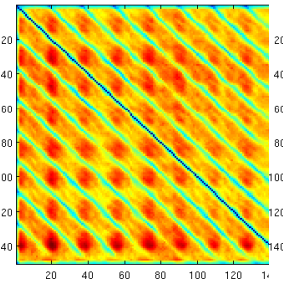

(b) SHvrS

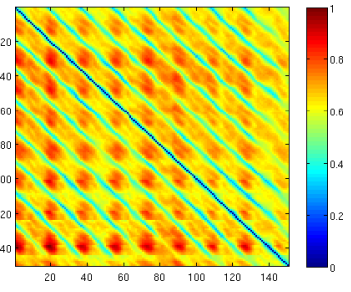

(c) SHvrG
Figure 2: Similarity Matrix for the motion runcircle. (a) Time filtered similarity matrix using a time window with size 5, $N_{t}=2$; (b) Similarity Matrix obtained by the shape-flow descriptor, the single-alignment SHvr (SHvrS), $N_{t}=2 ;$ (c) Similarity Matrix by the shape-flow descriptor, the groupalignment SHvr (SHvrG), $N_{t}=2$.

the descriptors. Let $X_{i}$ and $Y_{j}$ be $3 \mathrm{D}$ shape descriptors of individual frames in two motions, $d\left(X_{i}(\theta, \phi), Y_{j}(0,0)\right)$ the similarity distance with rotation $(\theta, \phi)$ using one of 3D shape descriptors, and $S(i, j)$, the similarity matrix. For a singleframe shape-flow matching, similarity is computed as follows:

$$
\begin{gathered}
\left(\theta_{\text {min }}, \phi_{\text {min }}\right)=\min _{\theta, \phi}\left\{d\left(X_{i}(\theta, \phi), Y_{j}(0,0)\right)\right\} \\
S_{\text {single }}(i, j)=\frac{\sum_{k=-N_{t}}^{k=N_{t}} d\left(X_{i+k}\left(\theta_{\text {min }}, \phi_{\text {min }}\right), Y_{j+k}(0,0)\right)}{2 N_{t}+1}
\end{gathered}
$$

where $\phi$ is $\mathrm{z}$-axis and $\theta$ is the rotation about the $\mathrm{z}$-axis, $\left(\theta_{\min }, \phi_{\min }\right)$ is found as the optimal rotation which minimises the distance for a pair of centre frames and $(0,0)$ denotes no rotation applied. For group-frame shape-flow matching, the optimal rotation is found by searching for the rotation that minimises the distance between two subsequences as a whole:

$$
\begin{gathered}
\left(\theta_{\text {min }}, \phi_{\text {min }}\right)=\min _{\theta, \phi}\left\{\frac{\sum_{k=-N_{t}}^{k=N_{t}} d\left(X_{i+k}(\theta, \phi), Y_{j+k}(0,0)\right)}{2 N_{t}+1}\right\} \\
S_{\text {group }}(i, j)=\frac{\sum_{k=-N_{t}}^{k=N_{t}} d\left(X_{i+k}\left(\theta_{\text {min }}, \phi_{\text {min }}\right), Y_{j+k}(0,0)\right)}{2 N_{t}+1}
\end{gathered}
$$

In practice, rotation is tested at a $1^{\circ}$ histogram resolution. The effect of the new descriptor is now illustrated in Figure 2. Figure 2 (b) and (c) show that the single-frame shape-flow similarity and the group-frame similarity are more distinctive than (a) time filtered similarity, reducing the ambiguity in matching forward and reverse motions in the periodic sequence.

\section{Experimental Evaluation}

A quantitative comparison is now presented for 3D Shape Descriptors with and without time-filtering in comparison to the new shape-flow descriptor using single-frame alignment and group-frame alignment. The receiver-operator characteristic (ROC) curve is used to show the relative performance of different methods. The synthetic ground-truth data-set is presented first, the different techniques are then compared. 

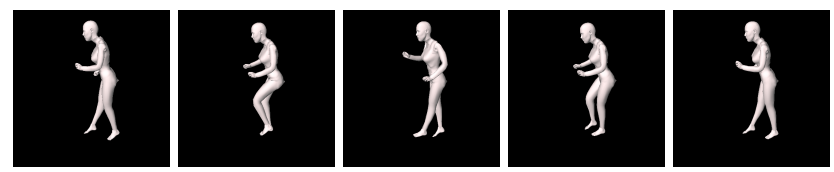

(a) Sneak
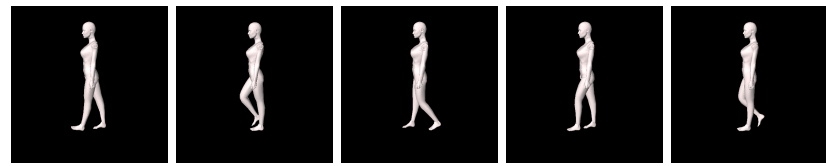

(c) Walk
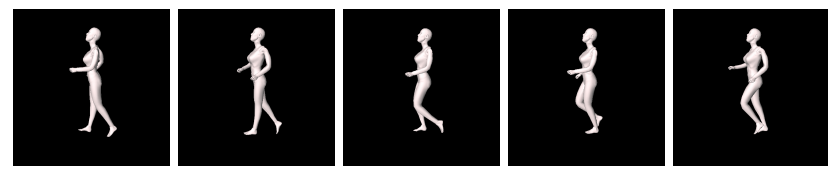

(e) Slow Run
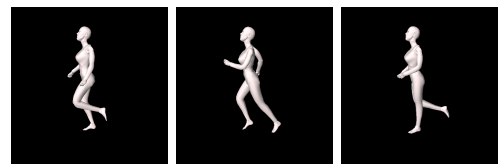

(g) Fast Run
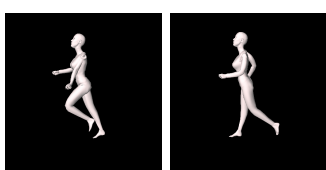
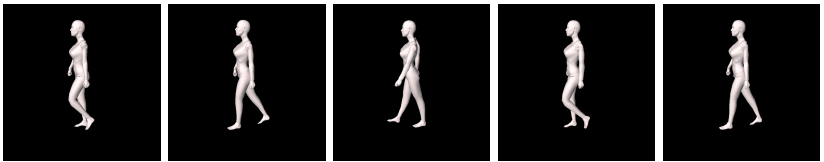

(b) Slow Walk
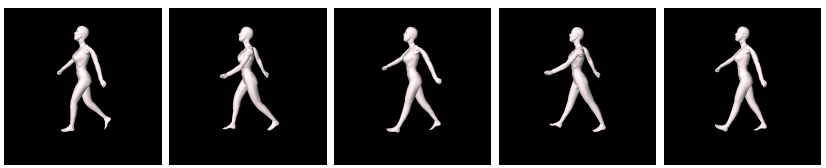

(d) Fast walk
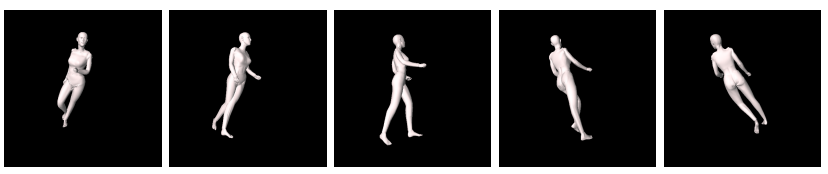

(f) Run Circle
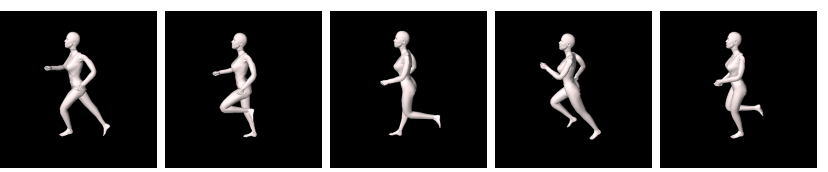

(h) Sprint

Figure 3: Simulated Data. (a) Sneak (b) Slow Walk (c) Walk (d) Fast Walk (e) Slow Run (f) Run Circle (g) Fast Run (h) Sprint at frame 1,16,31,46,61.

\subsection{Ground Truth}

A simulated data-set was constructed from a surface mesh with $20 \mathrm{k}$ vertices and $35 \mathrm{k}$ triangles animated using motion capture data. The data-set consisted of the following motions, with 150 frames per motion: sneak, slow walk, walk, fast walk, slow run, run circle, fast run, and sprint. Figure 3 shows some examples. Surface correspondence in the mesh is used only to generate ground-truth similarity, and not used to compute shape similarity measures. The ground-truth temporal similarity between two frames is defined by a combination of position similarity and velocity similarity. A rigid-body registration is performed to align frames for similarity assessment. Position similarity is then computed by finding the average vertex-to-corresponding-vertex distance. To compute velocity similarity, we first compute velocity for each vertex stored as a vector and then find the average vector distance between two surfaces. Let $X$ and $Y$ be the surface, each with $N$ vertices, if $d\left(x_{i}, y_{i}\right)$ denotes the Euclidean Distance between vertex $x_{i} \in X$ and its correspondent vertex $y_{i} \in Y, i=0,1, . ., N$, the position similarity measure is calculated as follows:

$$
P(X, Y)=\frac{1}{N} \sum_{i}\left\{d\left(x_{i}, y_{i}\right)\right\}
$$

Velocity similarity is then calculated at $t$ as:

$$
V(X, Y)=\frac{1}{N} \sum_{i}\left\{d_{v}\left(m_{i}, n_{i}\right)\right\}
$$

where $m_{i}=\frac{x_{i}(t+1)-x_{i}(t-1)}{2}, n_{i}=\frac{y_{i}(t+1)-y_{i}(t-1)}{2}$ are velocity vectors, $t+1, t-1$ denotes next and previous frame, and $d_{v}\left(m_{i}, n_{i}\right)=\left|m_{i}-n_{i}\right|$ is the magnitude of vector difference. A single similarity metric is defined by combination as follows:

$$
C(X, Y)=(1-\alpha) P(X, Y)+\alpha V(X, Y)
$$

Ground-truth matching is then defined by taking a single threshold $T$ for acceptable similarity. If $C(X, Y)<T$, similarity $S_{\text {true }}(X, Y)=1$ is defined, denoting that $X, Y$ are similar, otherwise, $S_{\text {true }}(X, Y)=0$ denoting that $X, Y$ are dissimilar. This process is illustrated in Figure 4, where position and velocity is combined and then thresholded to provide the ground-truth classification of similarity. Figure 5 shows the ground-truth classification for different motions.

\subsection{Comparison of Temporal 3D Shape Descriptors}

The performance of the different descriptors is now evaluated against the ground-truth classification of similarity.

\subsubsection{Evaluation of 3D Shape Descriptors}

The relative performance of the Shape Distribution (SD), Spin Image (SI), the rotated volume-spherical Shape Histogram (SHvr) and Spherical Harmonics Representation (SHR) descriptors is shown in Figure 6. For different descriptors, we choose best performing parameter settings [18]: SD, the number of samples, $N=1$ million; SI, the number of bins for $\alpha$ and $\beta$, $N_{\alpha}=N_{\beta}=40$; SHvr, the number of shells $N_{s}=10$, and the angular bins $N_{\phi}=0.5 * N_{\theta}=20$; SHR, the number of shells and the bandwidth, $N_{s}=N_{b w}=10$. 


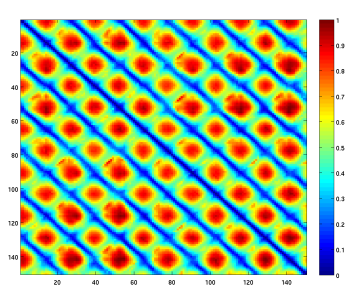

(a) Position

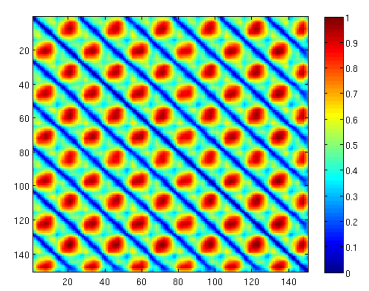

(b) Velocity

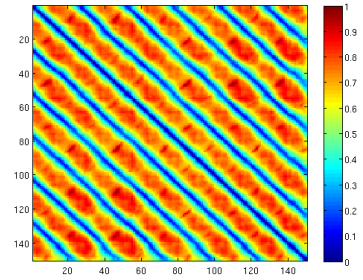

(c) Combination

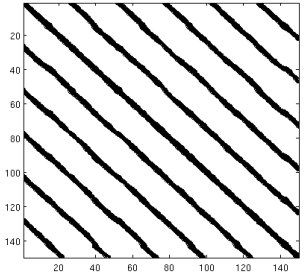

(d) Classification

Figure 4: Ground-Truth Generation. For slow run: (a) position similarity; (b) velocity similarity; (c) combination of position and velocity similarity; (d) classification after thresholding.

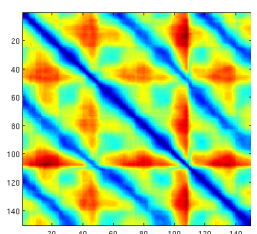

(a) Sneak

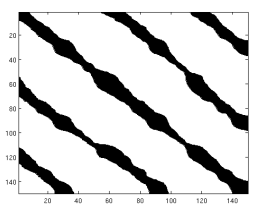

(g) Sneak

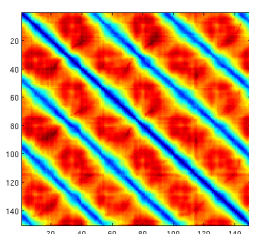

(b) Slow Walk

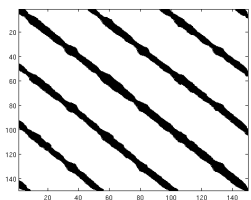

(h) Slow Walk

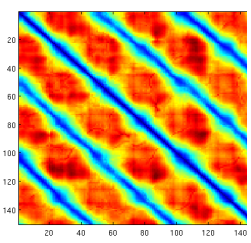

(c) Walk

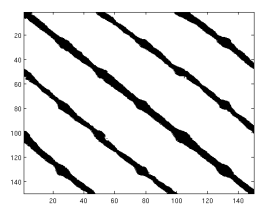

(i) Walk

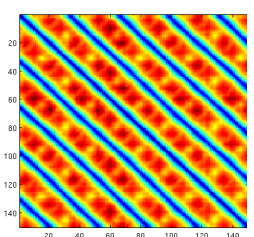

(d) Fast Walk

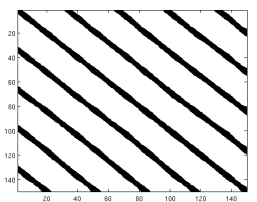

(j) Fast Walk

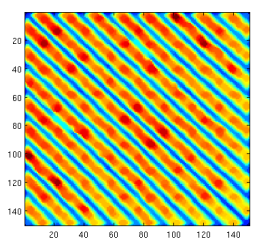

(e) Run Circle

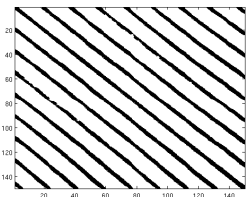

(k) Run Circle

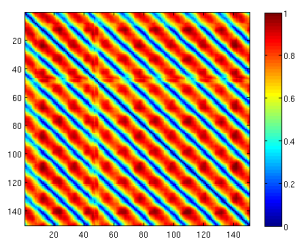

(f) Fast Run

Figure 5: Temporal Ground-truth Similarity (top) and Classification (bottom) for different motions including: (a,g) Sneak; (b,h) Slow Walk; (c,i) Walk; $(d, j)$ Fast Walk; (e,k) Run Circle; (f,l) Fast Run.

The results show that for each motion the SHvr descriptor outperforms the others. This result confirms previous work [18] in which this descriptor was demonstrated to have the greatest performance with a static ground-truth classification. SI descriptor performs well in most cases, in (f) for the motion run-circle the performance matches the SHvr. However the spin-image representation has a reflective ambiguity and so is not stable for all motions. $\mathrm{SD}$ is also invariant to a mirror transformation and so has an inherent ambiguity. SHR performs worst for this data-set due to the inherent "information loss" [16].

\subsubsection{Evaluation of Filtered Shape Descriptors}

Time-filtering incorporates temporal information by combining the static similarity scores over a time window. The performance increases slightly as shown in Figure 7 compared to Figure 6. For simplicity, we consider only a single window size of $3, N_{t}=1$. Again the volume-sampling spherical histogram SHvr demonstrates the highest performance compared to the other descriptors, with the exception of the motion run-circle in Figure 7(f).

\subsubsection{Evaluation of Shape-Flow Descriptors}

The rotated volume spherical Shape Histogram (SHvr) has been demonstrated to give the closest classification of shape similarity to the ground-truth data-set. The new shape-flow descriptor is now compared to the SHvr descriptor with and without using a time filter, again using a single time window $N_{t}=1$. Figure 8 (d-h) demonstrates that shape-flow outperforms both the static descriptor and temporal filtering. As the speed of the motion reduces from sprint to sneak, we can see that the performance of the descriptors converges and Figure 8 (a-c) shows little difference between the descriptors. This demonstrates that for slow motions the short window size does not incorporate sufficient variation to improve on the performance of the static descriptor.

The shape-flow descriptor is based on the local assumption that the changes in shape are consistent over the time-window for the subsequence in the descriptor. The choice of the window size depends on the specific motion. For example, with a slow motion, a larger window size is required to incorporate sufficient shape variation to discriminate between different motion subsequences. However, if the window is too large with fast motions the local assumption can be broken. The effect of window size is illustrated in Figure 9 for the run-circle motion. 


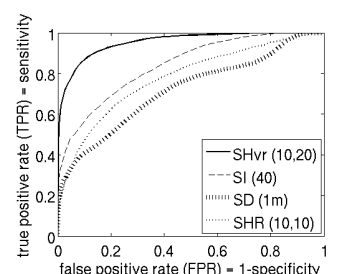

(a) Sneak

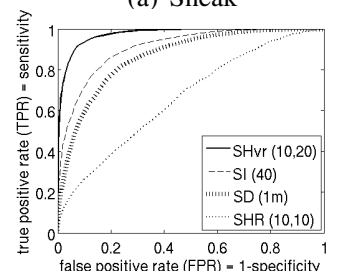

(e) Slow Run

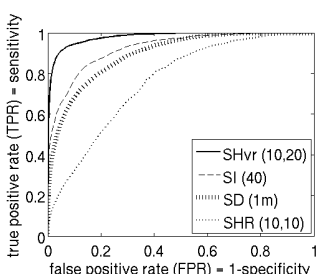

(b) Slow Walk

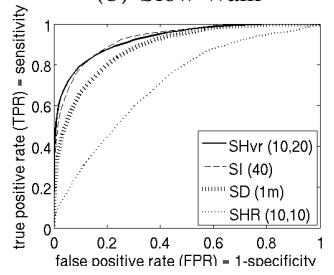

(f) Run Circle

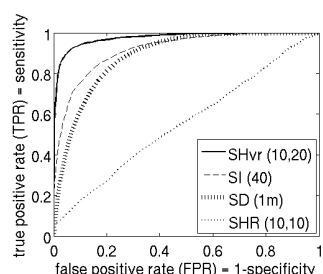

(c) Walk

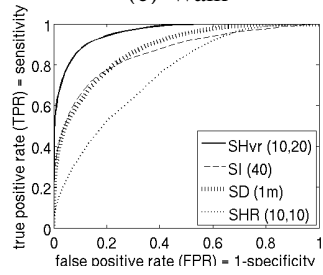

(g) Fast Run

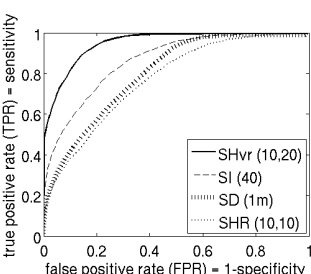

(d) Fast Walk

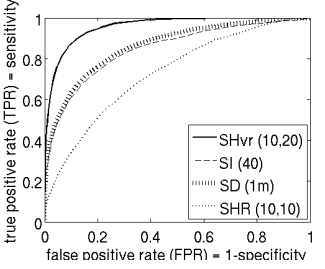

(h) Sprint

Figure 6: Evaluation for Shape Descriptors. ROC performance for sequences: (a) sneak; (b) slow walk; (c) walk; (d) fast walk; (e) slow run; (f) run circle; (g) fast run; (h) sprint.

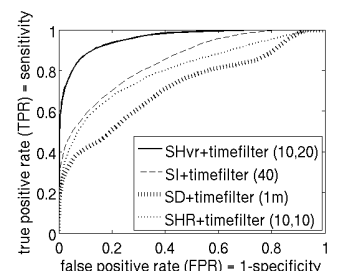

(a) Sneak

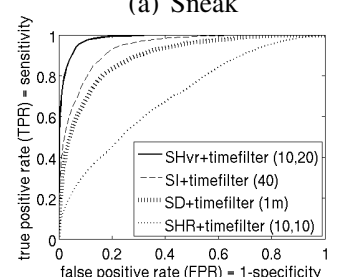

(e) Slow Run

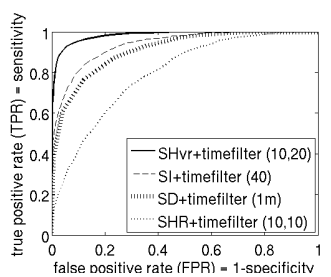

(b) Slow Walk

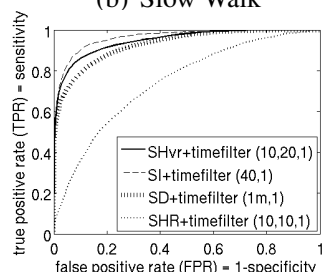

(f) Run Circle

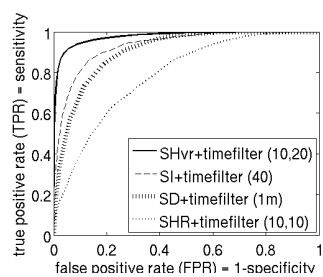

(c) Walk

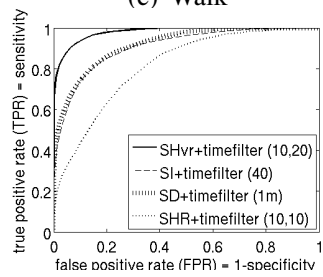

(g) Fast Run

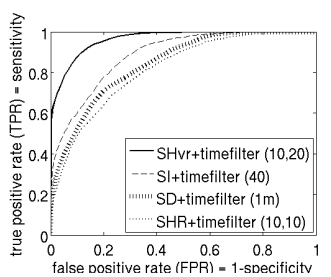

(d) Fast Walk

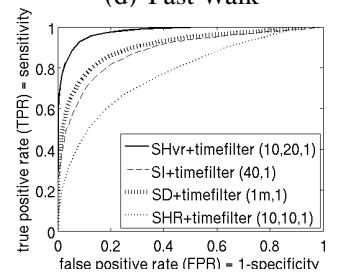

(h) Sprint

Figure 7: Evaluation for Shape Descriptors using a time filter. ROC performance for sequences: (a) sneak; (b) slow walk; (c) walk; (d) fast walk; (e) slow run; (f) run circle; (g) fast run; (h) sprint.

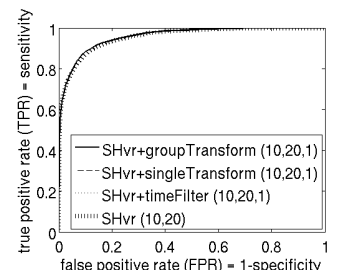

(a) Sneak

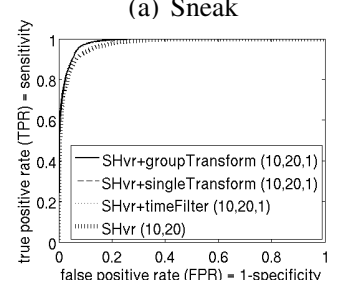

(e) Slow Run

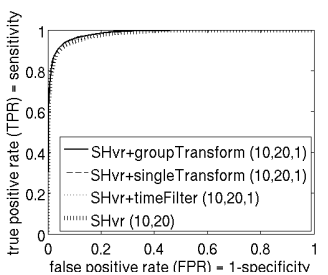

(b) Slow Walk

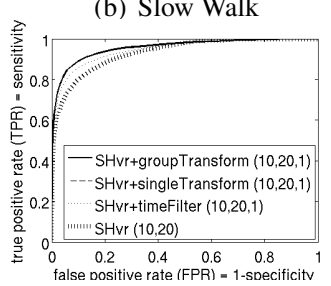

(f) Run Circle

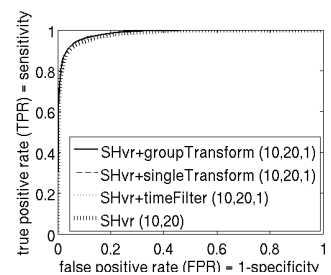

(c) Walk

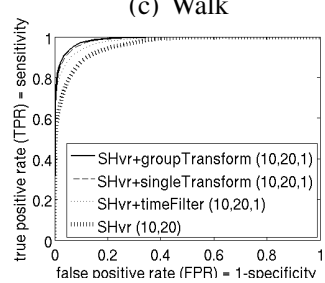

(g) Fast Run

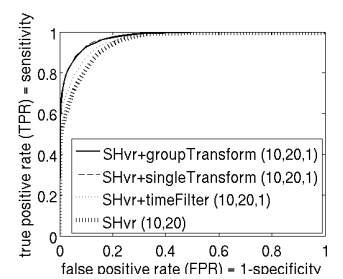

(d) Fast Walk

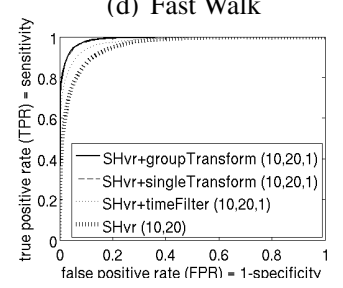

(h) Sprint

Figure 8: Evaluation for Shape-Flow Descriptors, $N_{t}=1$, ROC performance for sequences: (a) sneak; (b) slow walk; (c) walk; (d) fast walk; (e) slow run; (f) run circle; (g) fast run; (h) sprint. 


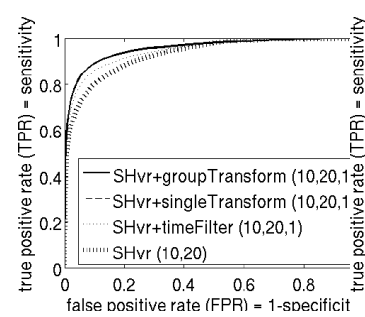

(a) $N_{t}=1$

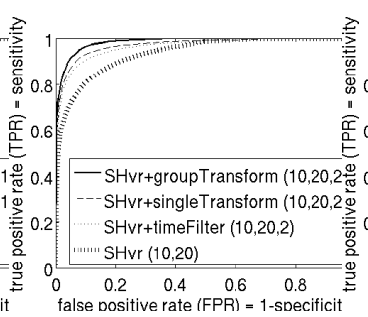

(b) $N_{t}=2$

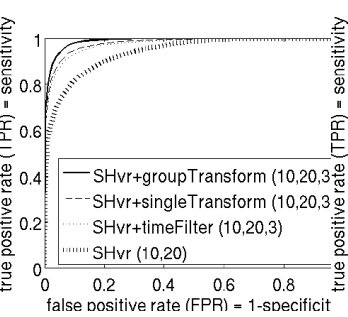

(c) $N_{t}=3$

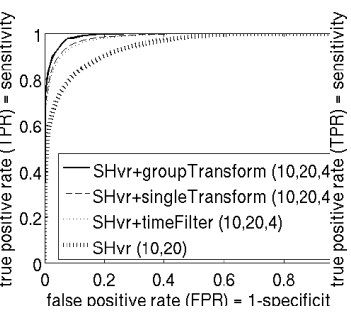

(d) $N_{t}=4$

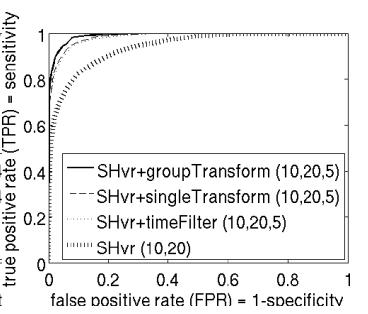

(e) $N_{t}=5$

Figure 9: Evaluation for Shape-Flow Descriptors using different window size. ROC performance for motion run circle: (a) $N_{t}=1 ;$ (b) $N_{t}=2$; (c) $N_{t}=3$; (d) $N_{t}=4$; (e) $N_{t}=5$.

As the window size is increased from 3 to 11 frames, $N_{t}=1$ to $N_{t}=5$, the performance of the descriptors increases.

For a small window size $N_{t}=1$ in Figure 8(f) and Figure 9(a) the performance of the group-alignment and single-alignment shape-flow descriptor is comparable and greater than the SHvr descriptor with and without time-filtering. As the window size is increased, the performance of the group-alignment improves over the single alignment: the group-alignment performs slightly better than single-alignment shape-flow descriptors and demonstrates an improvement on the SHvr descriptor with and without time-filtering in Figure 9(b-e).

\subsection{Evaluation on Real Data}

In this section, we apply the new shape-flow descriptor using the group-alignment ( $\mathrm{SHvrG}$ ) to 3D surface sequences of a street-dancer from a public database [29]. Each mesh contains around $140 \mathrm{k}$ vertices and $280 \mathrm{k}$ triangles. For the shape histogram a resolution of $N_{s}=10, N_{\phi}=20, N_{\theta}=40$ and for the temporal window $N_{t}=1$ is used. Intra-motion selfsimilarity is considered for pop dancing and inter-motion crosssimilarity between pop and lock dancing. Figure 10 shows the similarity matrix along with the curve for a single row. The query shape corresponds to the row and the local minima on each curve are illustrated.

\subsection{Discussion}

Our future work will focus on how to apply this new shapeflow descriptor to find proper transitions among time-varying sequences of human motion and connect them to generate new animations. The choice of time window size is critical in this application. It depends on the specific motion sequences and animation requirements: a slow-changing motion may require a bigger window size to incorporate enough temporal shape variation but a fast-changing motion requires a small window size to ensure local similarity. The choice of alignments must also be considered. The shape-flow descriptor uses two alignment schemes: a single-alignment (SHvrS) and a groupalignment (SHvrG). Both demonstrate an improvement on conventional 3D shape descriptors with and without using a time filter. With an appropriate window size group-alignment outperforms single-alignment but with a greater computational cost. The trade-off between accuracy and computational complexity may therefore be considered.

\section{Conclusion}

In this paper, we have presented a quantitative evaluation of conventional shape descriptors with and without using a time filter against a temporal ground-truth data; a new 4D histogram volume-based descriptor is then introduced to improve temporal shape matching, and this is demonstrated on real time-varying sequences of human motion. While the evaluation is not exhaustive due to breadth of the literature on 3D shape similarity, the work clearly demonstrates the advantage of the new shape-flow descriptor in the context of temporal shape matching in surface sequences.

\section{Acknowledgements}

This work was funded by EPSRC Grant EP/E001351.

\section{References}

[1] M. Ankerst, G. Kastenmller, H. P. Kriegel, and T. Seidl. 3D shape histograms for similarity search and classification in spatial databases. Advances in Spatial Databases, 6th International Symposium, SSD'99, 1651:207-228, 1999.

[2] Serge Belongie, Jitendra Malik, and Jan Puzicha. Shape context: A new descriptor for shape matching and object recognition. In NIPS, pages 831-837, 2000.

[3] Moshe Blank, Lena Gorelick, Eli Shechtman, Michal Irani, and Ronen Basri. Actions as space-time shapes. In ICCV '05: Proceedings of the Tenth IEEE International Conference on Computer Vision, pages 1395-1402, Washington, DC, USA, 2005. IEEE Computer Society.

[4] Aaron F. Bobick and James W. Davis. The recognition of human movement using temporal templates. IEEE Trans. Pattern Anal. Mach. Intell., 23(3):257-267, March 2001.

[5] Benjamin Bustos, Daniel Keim, Dietmar Saupe, and Tobias Schreck. Content-based 3d object retrieval. Computer Graphics and Applications, IEEE, 27(4):2227, 2007.

[6] Benjamin Bustos, Daniel A. Keim, Dietmar Saupe, Tobias Schreck, and Dejan V. Vrani. Feature-based similarity search in $3 \mathrm{~d}$ object databases. ACM Comput. Surv., 37(4):345-387, December 2005. 

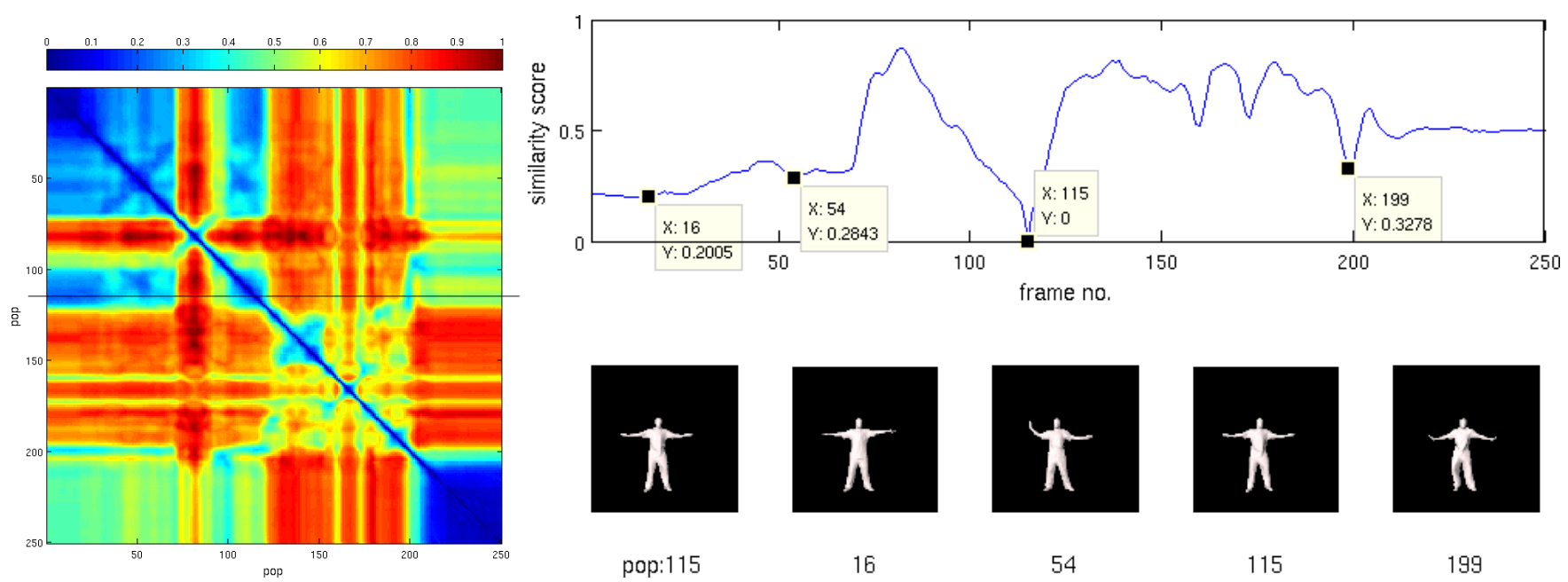

pop: 115

16

54

115

199

(a) Intra motion similarity for pop sequence
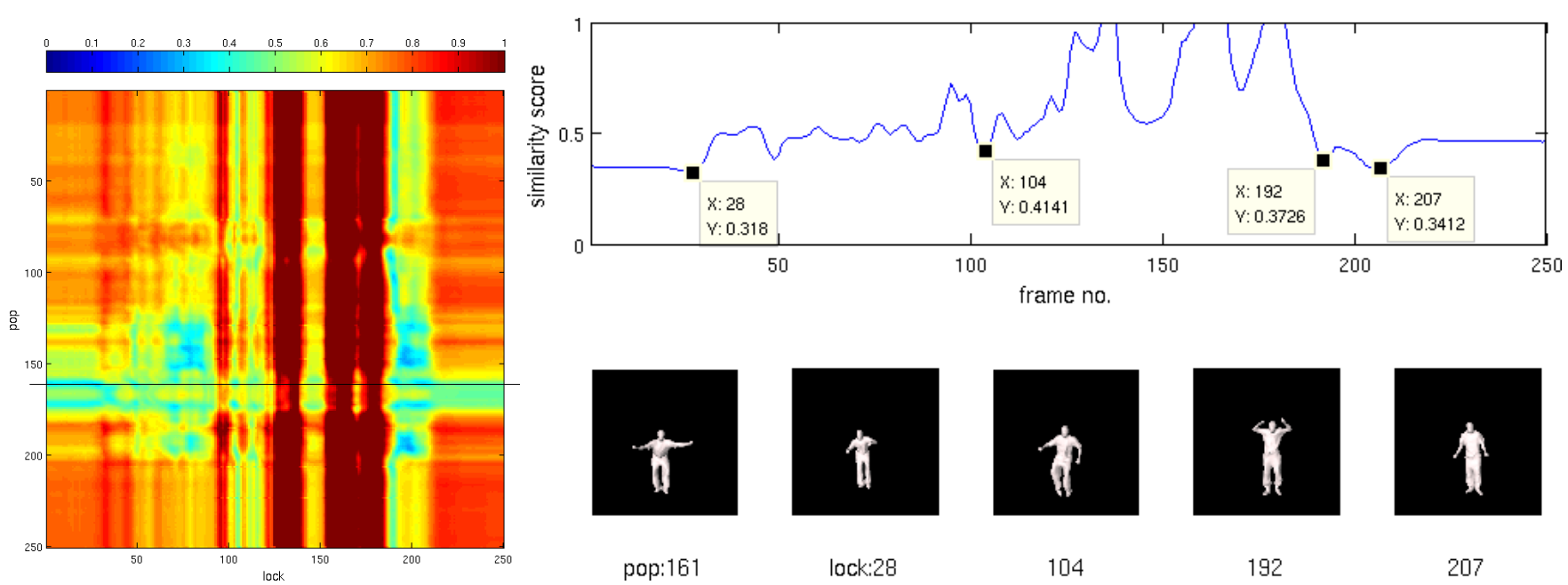

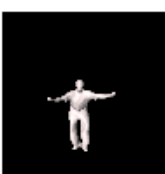

pop:161

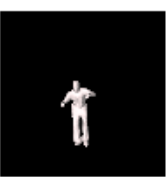

lock:28

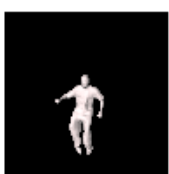

104

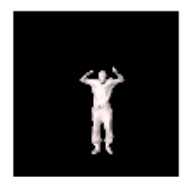

192

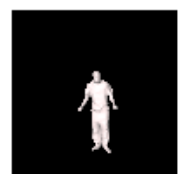

207

(b) Inter motion similarity between pop and lock sequence

Figure 10: Evaluation for SHvrG on Real Data, showing the similarity matrix and the similarity curve for a single row in the matrix. The shape for the row and local minima on the curves are illustrated.

[7] Chin S. Chua and Ray Jarvis. Point signatures: A new representation for $3 \mathrm{~d}$ object recognition. International Journal of Computer Vision, 25(1):63-85, October 1997.

[8] J. Corney, H. Rea, D. Clark, J. Pritchard, M. Breaks, and R. Macleod. Coarse filters for shape matching. Computer Graphics and Applications, IEEE, 22(3):65-74, 2002.

[9] Ross Cutler and Larry S. Davis. Robust real-time periodic motion detection, analysis, and applications. IEEE Trans. Pattern Anal. Mach. Intell., 22(8):781-796, August 2000.

[10] H. Dutagaci, B. Sankur, and Y. Yemez. Transform-based methods for indexing and retrieval of $3 \mathrm{~d}$ objects. pages 188-195, 2005.

[11] Alexei A. Efros, Alexander C. Berg, Greg Mori, and Jitendra Malik. Recognizing action at a distance. In ICCV '03: Proceedings of the Ninth IEEE International
Conference on Computer Vision, Washington, DC, USA, 2003. IEEE Computer Society.

[12] Thomas Funkhouser, Michael Kazhdan, Patrick Min, and Philip Shilane. Shape-based retrieval and analysis of $3 \mathrm{~d}$ models. Commun. ACM, 48(6):58-64, June 2005.

[13] Natraj Iyer, Subramaniam Jayanti, Kuiyang Lou, Yagnanarayanan Kalyanaraman, and Karthik Ramani. Three-dimensional shape searching: state-of-the-art review and future trends. Computer-Aided Design, 37(5):509-530, April 2005.

[14] A. E. Johnson and M. Hebert. Using spin images for efficient object recognition in cluttered 3d scenes. Pattern Analysis and Machine Intelligence, IEEE Transactions on, 21(5):433-449, 1999.

[15] Michael Kazhdan, Bernard Chazelle, David P. Dobkin, Adam Finkelstein, and Thomas A. Funkhouser. A 
reflective symmetry descriptor. In $E C C V$, volume 2, pages 642-656, 2002.

[16] Michael Kazhdan, Thomas Funkhouser, and Szymon Rusinkiewicz. Rotation invariant spherical harmonic representation of $3 \mathrm{~d}$ shape descriptors. $S G P$ '03: Proceedings of the 2003 Eurographics/ACM SIGGRAPH symposium on Geometry processing, pages 156-164, 2003.

[17] M. Kortgen, G. J. Park, M. Novotni, and R. Klein. 3d shape matching with $3 \mathrm{~d}$ shape contexts. In the 7th Central European Seminar on Computer Graphics, April 2003.

[18] Peng Huang, Jonathan Starck, and Adrian Hilton. A study of shape similarity for temporal surface sequences of people. In 3DIM 07, pages 408-418

[19] Peng Huang, Jonathan Starck, and Adrian Hilton. A quantitative comparison study of shape similarity. Technical report, 2007.

[20] M. Mousa, R. Chaine, and S. Akkouche. Direct spherical harmonic transform of a triangulated mesh. Journal of graphics tools, 11(2):17-26, 2006.

[21] Jan Neumann, Cornelia Fermller, and Yiannis Aloimonos. Animated heads: From 3d motion fields to action descriptions. In DEFORM 'OO/AVATARS 'O0: Proceedings of the IFIP TC5/WG5.10 DEFORM'2000 Workshop and AVATARS'2000 Workshop on Deformable Avatars, pages 1-11, Deventer, The Netherlands, The Netherlands, 2001. Kluwer, B.V.

[22] M. Novotni and R. Klein. 3d zernike descriptors for content based shape retrieval. The 8th ACM Symposium on Solid Modeling and Applications, June 2003.

[23] R. Ohbuchi, T. Minamitani, and T. Takei. Shapesimilarity search of $3 \mathrm{~d}$ models by using enhanced shape functions. Theory and Practice of Computer Graphics, 2003. Proceedings, pages 97-104, 2003.

[24] Robert Osada, Thomas Funkhouser, Bernard Chazelle, and David Dobkin. Shape distributions. ACM Trans. Graph., 21(4):807-832, October 2002.

[25] E. Paquet. Description of shape information for 2-d and 3-d objects. Sig. Proc.: Image Comm., 16:103-122, September 2000 .

[26] Julien Ricard, David Coeurjolly, and Atilla Baskurt. Art extension for description, indexing and retrieval of $3 \mathrm{~d}$ objects. In ICPR '04: Proceedings of the Pattern Recognition, 17th International Conference on (ICPR'04) Volume 3, pages 79-82, Washington, DC, USA, 2004. IEEE Computer Society.
[27] Arno Schodl, Richard Szeliski, David H. Salesin, and Irfan Essa. Video textures. In SIGGRAPH 'OO: Proceedings of the 27th annual conference on Computer graphics and interactive techniques, pages 489-498, New York, NY, USA, 2000. ACM Press/Addison-Wesley Publishing Co.

[28] Heung Y. Shum, Martial Hebert, and Katsushi Ikeuchi. On 3d shape similarity. Proceedings of the 1996 Conference on Computer Vision and Pattern Recognition (CVPR '96), pages 526-531, June 1996.

[29] J. Starck and A. Hilton. Surface capture for performancebased animation. IEEE Computer Graphics and Applications, 27(3):21-31, 2007.

[30] J. Starck, G. Miller, and A. Hilton. Video-based character animation. In SCA '05: Proceedings of the 2005 ACM SIGGRAPH/Eurographics symposium on Computer animation, pages 49-58, New York, NY, USA, 2005. ACM Press.

[31] J. W. H. Tangelder and R. C. Veltkamp. A survey of content based $3 \mathrm{~d}$ shape retrieval methods. Shape Modeling Applications, 2004. Proceedings, pages 145156, 2004.

[32] D. V. Vranic and D. Saupe. 3d shape descriptor based on 3d fourier transform. In Proceedings of the EURASIP Conference on Digital Signal Processing for Multimedia Communications and Services (ECMCS 2001) (editor K. Fazekas), pages 271-274, Budapest, Hungary, September 2001.

[33] Daniel Weinland, Remi Ronfard, and Edmond Boyer. Motion history volumes for free viewpoint action recognition. In IEEE International Workshop on modeling People and Human Interaction (PHI'05), 2005.

[34] Jianfeng Xu, Toshihiko Yamasaki, and Kiyoharu Aizawa. Motion editing in 3d video database. 3dpvt, 0:472-479, 2006.

[35] T. Zaharia and F. Preteux. Three-dimensional shapebased retrieval within the mpeg-7 framework. volume 4304, pages 133-145, January 2001.

[36] C. Zhang and T. Chen. Efficient feature extraction for $2 \mathrm{~d} / 3 \mathrm{~d}$ objects in mesh representation. pages 935-938, 2001 . 